Georgian Mathematical Journal

Volume 8 (2001), Number 2, 221-230

\title{
CONVERGENCE TO ZERO AND BOUNDEDNESS OF OPERATOR-NORMED SUMS OF RANDOM VECTORS WITH APPLICATION TO AUTOREGRESSION PROCESSES
}

\author{
V. V. BULDYGIN, V. A. KOVAL
}

\begin{abstract}
The problems of almost sure convergence to zero and almost sure boundedness of operator-normed sums of different sequences of random vectors are studied. The sequences of independent random vectors, orthogonal random vectors and the sequences of vector-valued martingale-differences are considered. General results are applied to the problem of asymptotic behaviour of multidimensional autoregression processes.
\end{abstract}

2000 Mathematics Subject Classification: Primary: 60F15. Secondary: 60G35, 60G42.

Key words and phrases: Operator-normed sums of random vectors, independent random vectors, orthogonal random vectors, vector-valued martingale-differences, almost sure convergence to zero, almost sure boundedness.

\section{INTRODUCTION}

The problems of almost sure convergence to zero and almost sure boundedness of sequences of random variables, and the interpretation of these problems from the viewpoint of probability in Banach spaces is comprised within the scope of scientific interests of N. N. Vakhania [1,2]. This paper deals with almost sure convergence to zero and almost sure boundedness of operator-normed sums of random vectors. In Section 2, a brief survey of the results related to almost sure convergence to zero for these sums is given. In Section 3, integral type conditions for almost sure convergence to zero and almost sure boundedness of operatornormed sums of independent random vectors are obtained. In Section 4, these results are applied to the study of asymptotic behaviour of multidimensional autoregression processes.

We introduce the following notation: $\mathbb{R}^{n}$ is $n$-dimensional Euclidean space; $\left(A_{n}, n \geq 1\right) \subset \mathcal{L}\left(\mathbb{R}^{m}, \mathbb{R}^{d}\right)$, where $\mathcal{L}\left(\mathbb{R}^{m}, \mathbb{R}^{d}\right)$ is the class of all linear operators (matrices) mapping $\mathbb{R}^{m}$ into $\mathbb{R}^{d},(m, d \geq 1) ;\|x\|$ and $\langle x, y\rangle$ are the norm of the vector $x$ and the inner product of the vectors $x$ and $y$ correspondingly; $\|A\|=\sup _{\|x\|=1}\|A x\|$ is the norm of the operator (matrix) $A ; A^{*}\left(A^{\top}\right)$ is the conjugate operator (matrix) of $A ; \mathfrak{N}_{\infty}$ is the class of all strictly monotone infinite sequences of positive integers; $\left(X_{k}, k \geq 1\right)$ is a sequence of random vectors in $\mathbb{R}^{m}$ defined on a probability space $\{\Omega, \mathcal{F}, \mathbf{P}\} ; S_{n}=\sum_{k=1}^{n} X_{k}, n \geq 1 ; 1\{\mathcal{D}\}$ is

ISSN 1072-947X / \$8.00 / C) Heldermann Verlag www.heldermann.de 
the indicator of $\mathcal{D} \in \mathcal{F} ; \sum_{i=n+1}^{n}(\cdot)=0$. Recall that a random vector $X$ is called symmetric if $X$ and $-X$ are identically distributed.

\section{Almost Sure Convergence to Zero of Operator-Normed Sums OF RANDOM VECTORS}

Prokhorov-Loève type conditions for almost sure convergence to zero of operator-normed sums of independent random vectors. First we consider the case where $\left(X_{k}, k \geq 1\right)$ is a sequence of independent random vectors.

Theorem 1 ([3]). Let $\left(X_{k}, k \geq 1\right)$ be a sequence of independent symmetric random vectors. Assume that $\left\|A_{n} S_{n}\right\| \underset{n \rightarrow \infty}{\longrightarrow} 0$ almost surely. Then:

(i) for any $k \geq 1$

$$
\left\|A_{n} X_{k}\right\| \underset{n \rightarrow \infty}{\longrightarrow} 0 \text { almost surely; }
$$

(ii) for any sequence $\left(n_{j}, j \geq 1\right) \in \mathfrak{N}_{\infty}$

$$
\left\|A_{n_{j+1}}\left(S_{n_{j+1}}-S_{n_{j}}\right)\right\| \underset{j \rightarrow \infty}{\longrightarrow} 0 \text { almost surely. }
$$

Theorem 2 ([3]). Let $\left(X_{k}, k \geq 1\right)$ be a sequence of independent symmetric random vectors. Assume that:

(i) for any $k \geq 1$

$$
\left\|A_{n} X_{k}\right\| \underset{n \rightarrow \infty}{\longrightarrow} 0 \text { in probability }
$$

Then there exists a finite class $\mathfrak{N}_{f} \subset \mathfrak{N}_{\infty}$ depending on the sequence $\left(A_{n}, n \geq 1\right)$ only, such that, given that the condition

(ii)

$$
\left\|A_{n_{j+1}}\left(S_{n_{j+1}}-S_{n_{j}}\right)\right\| \underset{j \rightarrow \infty}{\longrightarrow} 0 \quad \text { almost surely }
$$

holds for all $\left(n_{j}, j \geq 1\right) \in \mathfrak{N}_{f}$, one has

$$
\left\|A_{n} S_{n}\right\| \underset{n \rightarrow \infty}{\longrightarrow} 0 \text { almost surely. }
$$

Remark. Let $\left(X_{k}, k \geq 1\right)$ be a sequence of independent random vectors which need not be symmetric. If assumptions (i) and (ii) in Theorem 2 hold and if $\left\|A_{n} S_{n}\right\| \rightarrow 0$ in probability as $n \rightarrow \infty$, then (2) holds.

Almost sure convergence to zero of operator-normed sums of orthogonal random vectors. Now consider the case where $\left(X_{k}, k \geq 1\right)$ is a sequence of orthogonal random vectors. By definition, this means that $\mathbf{E}\left\|X_{k}\right\|^{2}<$ $\infty, k \geq 1$, and for any $a \in \mathbb{R}^{m}$ and $j \neq k \quad \mathbf{E}\left(\left\langle a, X_{j}\right\rangle\left\langle a, X_{k}\right\rangle\right)=0$.

Theorem 3 ([4]). Let $\left(X_{k}, k \geq 1\right)$ be a sequence of orthogonal random vectors. Assume that condition (1) holds. Then there exists a finite class $\mathfrak{N}_{f} \subset$ 
$\mathfrak{N}_{\infty}$ depending on the sequence $\left(A_{n}, n \geq 1\right)$ only, such that, given that the condition

$$
\sum_{j=1}^{\infty} \sum_{k=n_{j}+1}^{n_{j+1}} \mathbf{E}\left\|A_{n_{j+1}} X_{k}\right\|^{2}\left(\log 4\left(n_{j+1}-n_{j}\right)\right)^{2}<\infty
$$

holds for all $\left(n_{j}, j \geq 1\right) \in \mathfrak{N}_{f}$, one has (2).

Corollary 1. Let $\left(X_{k}, k \geq 1\right)$ be a sequence of orthogonal random vectors. Assume that $\left\|A_{n}\right\| \rightarrow 0$ as $n \rightarrow \infty$ and suppose that

$$
\sum_{k=1}^{\infty} \sup _{n \geq k}\left(\mathbf{E}\left\|A_{n} X_{k}\right\|^{2} \log ^{2} n\right)<\infty
$$

Then (2) holds.

Almost sure convergence to zero of operator-normed vector-valued martingales. Let $\left(S_{n}, \mathcal{F}_{n}, n \geq 0\right), S_{0}=0$, be a martingale in $\mathbb{R}^{m}$, that is $X_{k}=S_{k}-S_{k-1}, k \geq 1$, is a martingale-difference in $\mathbb{R}^{m}$.

Theorem 4 ([5]). Let $\left(X_{k}, k \geq 1\right)$ be a martingale-difference. Assume that condition (1) holds. Then there exists a finite class $\mathfrak{N}_{f} \subset \mathfrak{N}_{\infty}$ depending on the sequence $\left(A_{n}, n \geq 1\right)$ only, such that, given that the condition

$$
\sum_{j=1}^{\infty} \mathbf{E}\left(\left\|A_{n_{j+1}}\left(S_{n_{j+1}}-S_{n_{j}}\right)\right\| 1\left\{\left\|A_{n_{j+1}}\left(S_{n_{j+1}}-S_{n_{j}}\right)\right\|>\varepsilon\right\}\right)<\infty,
$$

or equivalently, given the condition

$$
\sum_{j=1}^{\infty} \int_{\varepsilon}^{\infty} \mathbf{P}\left\{\left\|A_{n_{j+1}}\left(S_{n_{j+1}}-S_{n_{j}}\right)\right\|>t\right\} d t<\infty
$$

holds for all $\left(n_{j}, j \geq 1\right) \in \mathfrak{N}_{f}$ and any $\varepsilon>0$, one has $(2)$.

Theorem 5 ([5, 6]). Let $\left(X_{k}, k \geq 1\right)$ be a martingale-difference; $p>1$, and $\mathbf{E}\left\|X_{k}\right\|^{p}<\infty, k \geq 1$. Assume that condition (1) holds. Then there exists a finite class $\mathfrak{N}_{f} \subset \mathfrak{N}_{\infty}$ depending on the sequence $\left(A_{n}, n \geq 1\right)$ only, such that, given that the condition

$$
\sum_{j=1}^{\infty} \mathbf{E}\left\|A_{n_{j+1}}\left(S_{n_{j+1}}-S_{n_{j}}\right)\right\|^{p}<\infty
$$

holds for all $\left(n_{j}, j \geq 1\right) \in \mathfrak{N}_{f}$, one has (2).

Corollary 2. Let $\left(X_{k}, k \geq 1\right)$ be a martingale-difference; $p \in(1,2]$, and $\mathbf{E}\left\|X_{k}\right\|^{p}<\infty, k \geq 1$. Assume that condition (1) holds. Then there exists a finite class $\mathfrak{N}_{f} \subset \mathfrak{N}_{\infty}$ depending on the sequence $\left(A_{n}, n \geq 1\right)$ only, such that, given that the condition

$$
\sum_{j=1}^{\infty} \sum_{k=n_{j}+1}^{n_{j+1}} \mathbf{E}\left\|A_{n_{j+1}} X_{k}\right\|^{p}<\infty
$$

holds for all $\left(n_{j}, j \geq 1\right) \in \mathfrak{N}_{f}$, one has (2). 
Corollary 3. Let $\left(X_{k}, k \geq 1\right)$ be a martingale-difference; $p \in(1,2]$, and $\mathbf{E}\left\|X_{k}\right\|^{p}<\infty, k \geq 1$. Assume that condition (1) holds. If

$$
\sum_{k=1}^{\infty} \sup _{n \geq k}\left\|A_{n} X_{k}\right\|^{p}<\infty
$$

then (2) holds.

Corollary 3 implies a result due to Kaufmann [7].

Corollary 4. Let $\left(X_{k}, k \geq 1\right)$ be a martingale-difference; $p \in(1,2]$, and $\mathbf{E}\left\|X_{k}\right\|^{p}<\infty, k \geq 1$. Assume that $\left\|A_{n}\right\| \rightarrow 0$ as $n \rightarrow \infty$ and $\left\|A_{n} x\right\| \geq\left\|A_{n+1} x\right\|$ for all $x \in \mathbb{R}^{m}, n \geq 1$. If

then (2) holds.

$$
\sum_{k=1}^{\infty}\left\|A_{k} X_{k}\right\|^{p}<\infty
$$

Example. Consider the assumption leading to strong consistency of the least squares estimator $\hat{\theta}_{n}, n \geq 1$, of an unknown parameter $\theta \in \mathbb{R}^{m}$ in the multivariate linear regression model $Y_{k}=B_{k} \theta+Z_{k}, k \geq 1$. Here $\left(Z_{k}, k \geq 1\right)$ is a martingale-difference in $\mathbb{R}^{d} ;\left(B_{k}, k \geq 1\right) \subset \mathcal{L}\left(\mathbb{R}^{m}, \mathbb{R}^{d}\right)$. Since

$$
\hat{\theta}_{n}=\theta+\left(\sum_{k=1}^{n} B_{k}^{\top} B_{k}\right)^{-1} \sum_{k=1}^{n} B_{k}^{\top} Z_{k}, \quad n \geq 1,
$$

taking $p=2$ in Theorem 5 implies a result due to Lai [8]:

If $\sup _{k \geq 1} \mathbf{E}\left\|Z_{k}\right\|^{2}<\infty$ and if $\left\|\left(\sum_{k=1}^{n} B_{k}^{\top} B_{k}\right)^{-1}\right\| \rightarrow 0$ as $n \rightarrow \infty$, then $\left\|\hat{\theta}_{n}-\theta\right\| \rightarrow 0$ almost surely as $n \rightarrow \infty$.

Almost sure convergence to zero of operator-normed sub-Gaussian vector-valued martingales. A stochastic sequence $\left(Z_{k}, k \geq 1\right)=\left(Z_{k}, \mathcal{F}_{k}, k \geq\right.$ $1)$ is called a sub-Gaussian martingale-difference in $\mathbb{R}^{m}$, if: 1) $\left(Z_{k}, \mathcal{F}_{k}, k \geq 1\right)$ is a martingale-difference in $\left.\mathbb{R}^{m} ; 2\right)$ for any $k \geq 1$ the random vector $Z_{k}=$ $\left(Z_{k 1}, \ldots, Z_{k m}\right)^{\top}$ is conditionally sub-Gaussian with respect to the $\sigma$-algebra $\mathcal{F}_{k-1}$. Assumption 2) means that $\tau\left(Z_{k j}\right)<\infty$ for any $j=1, \ldots, m$ and any $k \geq 1$. Here

$$
\tau\left(Z_{k j}\right)=\inf \left\{a \geq 0: \mathbf{E}_{\mathcal{F}_{k-1}} e^{u Z_{k j}} \leq e^{a^{2} u^{2} / 2} \quad \text { almost surely, } u \in \mathbb{R}\right\} .
$$

We write $\tau\left(Z_{k}\right)=\max _{j=1, \ldots, m} \tau\left(Z_{k j}\right), k \geq 1$.

Theorem 6. Let $X_{k}=B_{k} Z_{k}, k \geq 1$, where $\left(Z_{k}, k \geq 1\right)$ is a sub-Gaussian martingale-difference in $\mathbb{R}^{m}$, $\sup _{k \geq 1} \tau\left(Z_{k}\right)<\infty$ and let $\left(B_{k}, k \geq 1\right) \subset \mathcal{L}\left(\mathbb{R}^{m}, \mathbb{R}^{m}\right)$. Assume that condition (1) holds. Then there exists a finite class $\mathfrak{N}_{f} \subset \mathfrak{N}_{\infty}$ depending on the sequence $\left(A_{n}, n \geq 1\right)$ only, such that, given that the condition

$$
\sum_{j=1}^{\infty} \exp \left(-\varepsilon\left(\sum_{k=n_{j}+1}^{n_{j+1}}\left\|A_{n_{j+1}} B_{k}\right\|^{2}\right)^{-1}\right)<\infty
$$

holds for all $\left(n_{j}, j \geq 1\right) \in \mathfrak{N}_{f}$ and any $\varepsilon>0$, one has (2). 
Remark. If $\left(Z_{k}, k \geq 1\right)$ is a sequence of independent Gaussian vectors, then conditions (1) and (3) are necessary for (2), see [3].

\section{Almost Sure Convergence to Zero and Almost Sure BOUNDEDNESS OF OpERATOR-NORMED SuMs OF INDEPENDENT RANDOM VECTORS}

Theorem 7. Let $\left(X_{k}, k \geq 1\right)$ be a sequence of independent zero-mean random vectors. Assume that

$$
\left\|A_{n}\right\| \underset{n \rightarrow \infty}{\longrightarrow} 0
$$

and for any sequence $\left(n_{j}, j \geq 1\right) \in \mathfrak{N}_{\infty}$ there exists $\delta \in(0,1]$ such that

$$
\sum_{j=1}^{\infty} \sum_{k=n_{j}+1}^{n_{j+1}} \mathbf{E}\left\|A_{n_{j+1}} X_{k}\right\|^{2+\delta}<\infty .
$$

If for any sequence $\left(n_{j}, j \geq 1\right) \in \mathfrak{N}_{\infty}$ and all $\varepsilon>0$

$$
\sum_{j=1}^{\infty} \exp \left(-\varepsilon\left(\sum_{k=n_{j}+1}^{n_{j+1}} \mathbf{E}\left\|A_{n_{j+1}} X_{k}\right\|^{2}\right)^{-1}\right)<\infty,
$$

then (2) holds.

If $X_{k}, k \geq 1$, are symmetric random vectors, then this theorem follows from Theorem 4.1.1 in [3]. Now we consider an independent proof in general case. To prove the theorem, we need the following lemma [9].

Lemma 1. Let $\left(\xi_{1}, \ldots, \xi_{n}\right)$ be independent random variables such that $\mathbf{E}\left|\xi_{i}\right|^{2+\delta}<\infty, 1 \leq i \leq n$, for some $\delta \in(0,1]$. Let $\eta$ be a zero-mean Gaussian random variable such that $\mathbf{E} \eta^{2}=\sum_{i=1}^{n} \mathbf{E} \xi_{i}^{2}$. Then for any $t>0$

$$
\left|\mathbf{P}\left(\left|\sum_{i=1}^{n} \xi_{i}\right|>t\right)-\mathbf{P}(|\eta|>t)\right| \leq \frac{c}{t^{2+\delta}} \sum_{i=1}^{n} \mathbf{E}\left|\xi_{i}\right|^{2+\delta}
$$

where $c$ is an absolute constant.

Proof of Theorem 7 . Let $\left(e_{1}, \ldots, e_{d}\right)$ be an orthonormal basis in $\mathbb{R}^{d}$. Then

$$
A_{n} S_{n}=\sum_{k=1}^{d}\left\langle A_{n}^{*} e_{k}, S_{n}\right\rangle e_{k}, \quad n \geq 1 .
$$

Therefore the theorem will be proved if we show that

$$
\left\langle A_{n}^{*} e, S_{n}\right\rangle \underset{n \rightarrow \infty}{\longrightarrow} 0 \quad \text { almost surely }
$$

for an arbitrary fixed vector $e \in \mathbf{R}^{d},\|e\|=1$. Since by (4)

$$
\left\|A_{n}^{*} e\right\| \underset{n \rightarrow \infty}{\longrightarrow} 0
$$


and since $\left(S_{n}, n \geq 1\right)$ is a martingale, it is sufficient to show, by Theorem 4 , that for all $\left(n_{j}, j \geq 1\right) \in \mathfrak{N}_{\infty}$ and any $\varepsilon>0$

$$
\sum_{j=1}^{\infty} \int_{\varepsilon}^{\infty} \mathbf{P}\left(\left|\sum_{k=n_{j}+1}^{n_{j+1}}\left\langle A_{n_{j+1}}^{*} e, X_{k}\right\rangle\right|>t\right) d t<\infty .
$$

By Lemma 1 , for any $j \geq 1$

$$
\begin{aligned}
\mathbf{P}\left(\mid \sum_{k=n_{j}+1}^{n_{j+1}}\right. & \left.\left\langle A_{n_{j+1}}^{*} e, X_{k}\right\rangle \mid>t\right) \\
& \leq \mathbf{P}\left(\left|\eta_{j}\right|>t\right)+\frac{c}{t^{2+\delta}} \sum_{k=n_{j}+1}^{n_{j+1}} \mathbf{E}\left|\left\langle A_{n_{j+1}}^{*} e, X_{k}\right\rangle\right|^{2+\delta} \\
& \leq \mathbf{P}\left(\left|\eta_{j}\right|>t\right)+\frac{c}{t^{2+\delta}} \sum_{k=n_{j}+1}^{n_{j+1}} \mathbf{E}\left\|A_{n_{j+1}} X_{k}\right\|^{2+\delta}
\end{aligned}
$$

where $\eta_{j}$ is a normally distributed random variable with parameters

$$
\mathbf{E} \eta_{j}=0, \quad \mathbf{E} \eta_{j}^{2}=\sum_{k=n_{j}+1}^{n_{j+1}} \mathbf{E}\left\langle A_{n_{j+1}}^{*} e, X_{k}\right\rangle^{2}
$$

Since for all $t>0$

$$
\mathbf{P}\left(\left|\eta_{j}\right|>t\right) \leq 2 \exp \left(-\frac{t^{2}}{2 \mathbf{E} \eta_{j}^{2}}\right)
$$

and

$$
\mathbf{E} \eta_{j}^{2} \leq \sum_{k=n_{j}+1}^{n_{j+1}} \mathbf{E}\left\|A_{n_{j+1}} X_{k}\right\|^{2}
$$

condition (6) implies

$$
\begin{aligned}
\sum_{j=1}^{\infty} \int_{\varepsilon}^{\infty} \mathbf{P}\left(\left|\eta_{j}\right|>t\right) d t & \leq 4 \sqrt{2} \sum_{j=1}^{\infty}\left(\mathbf{E} \eta_{j}^{2}\right)^{\frac{1}{2}} \exp \left(-\frac{\varepsilon^{2}}{2 \mathbf{E} \eta_{j}^{2}}\right) \\
& \leq 4 \sqrt{2} \sup _{j \geq 1}\left(\mathbf{E} \eta_{j}^{2}\right)^{\frac{1}{2}} \sum_{j=1}^{\infty} \exp \left(-\frac{\varepsilon^{2}}{2 \mathbf{E} \eta_{j}^{2}}\right)<\infty
\end{aligned}
$$

Taking into account the inequalities (8) and (5) we conclude that (7) holds.

Theorem 8. Let $\left(X_{k}, k \geq 1\right)$ be a sequence of independent zero-mean random vectors. Assume that $\sup _{n \geq 1}\left\|A_{n}\right\|<\infty$ and that for any $\left(n_{j}, j \geq 1\right) \in \mathfrak{N}_{\infty}$ there exists $\delta \in(0,1]$ such that $(5)$ holds. If for any $\left(n_{j}, j \geq 1\right) \in \mathfrak{N}_{\infty}$ there exists $\varepsilon>0$ such that (6) holds, then

$$
\sup _{n \geq 1}\left\|A_{n} S_{n}\right\|<\infty \quad \text { almost surely. }
$$

Proof. Theorem 8 follows from Theorem 7 and Lemma 3 in [10]. We refer the reader to [3] for more details. 
Theorem 9. Let $\left(X_{k}, k \geq 1\right)$ be a sequence of independent zero-mean random vectors. Assume that $\left\|A_{n}\right\| \underset{n \rightarrow \infty}{\longrightarrow} 0$ and for any $\left(n_{j}, j \geq 1\right) \in \mathfrak{N}_{\infty}$ there exists $\delta \in(0,1]$ such that (5) holds. If for any $\left(n_{j}, j \geq 1\right) \in \mathfrak{N}_{\infty}$ there exists $\varepsilon>0$ such that (6) holds, then there exists a nonrandom constant $L \in[0, \infty)$ such that

$$
\limsup _{n \rightarrow \infty}\left\|A_{n} S_{n}\right\|=L \quad \text { almost surely. }
$$

Proof. Theorem 9 follows from Theorem 8 and Theorem 2.3.2 in [3]. We refer the reader to [3] for more details.

The next corollary of Theorem 9 can be useful in applications.

Corollary 5. Let $\left(X_{i}, i \geq 1\right)$ be a sequence of independent zero-mean random vectors. Assume that $\left\|A_{n}\right\| \underset{n \rightarrow \infty}{\longrightarrow} 0$ and suppose that there exists $\delta \in(0,1]$ such that the following condition holds

$$
\sum_{i=1}^{\infty} \sup _{n \geq i} \mathbf{E}\left\|A_{n} X_{i}\right\|^{2+\delta}<\infty .
$$

Assume also that there are two sequences of positive numbers $\left(\varphi_{n}, n \geq 1\right)$, $\left(f_{n}, n \geq 1\right)$, such that $\left(f_{n}, n \geq 1\right)$ is monotonically nondecreasing and the inequality

$$
\sum_{i=k+1}^{n} \mathbf{E}\left\|A_{n} X_{i}\right\|^{2} \leq \varphi_{n}\left[1-\left(\frac{f_{k}}{f_{n}}\right)^{2}\right]
$$

holds for all $n>k \geq 1$. If for some $M>0$

$$
\sup _{n \geq 2} \varphi_{n} \ln \left(2+\sum_{k=1}^{n-1} \min \left\{M, \ln \left(\frac{f_{k+1}}{f_{k}}\right)\right\}\right)<\infty,
$$

then there exists a nonrandom constant $L \in[0, \infty)$ such that (9) holds.

Proof. Let us show that (5) follows from (10):

$$
\begin{aligned}
\sum_{j=1}^{\infty} \sum_{i=n_{j}+1}^{n_{j+1}} \mathbf{E}\left\|A_{n_{j+1}} X_{i}\right\|^{2+\delta} & \leq \sum_{j=1}^{\infty} \sum_{i=n_{j}+1}^{n_{j+1}} \sup _{n \geq i} \mathbf{E}\left\|A_{n} X_{i}\right\|^{2+\delta} \\
& \leq \sum_{i=1}^{\infty} \sup _{n \geq i} \mathbf{E}\left\|A_{n} X_{i}\right\|^{2+\delta}<\infty
\end{aligned}
$$

In addition, for all $\left(n_{j}, j \geq 1\right) \in \mathfrak{N}_{\infty}$ and some $\varepsilon>0$ the condition (6) follows from (11) and (12), see [5]. 


\section{Asymptotic Behaviour of Multidimensional Autoregression PROCESSES}

Let us apply Corollary 5 for obtaining an analog of the bounded law of iterated logarithm for autoregressive processes. Let $\mathbb{R}^{m}$ be a space of column vectors. Consider an autoregression equation in $\mathbb{R}^{m}$ written as follows:

$$
Y_{n}=A Y_{n-1}+V_{n}, \quad n \geq 1, Y_{0} \in \mathbb{R}^{m} .
$$

Here $A$ is an arbitrary $m \times m$ matrix and $\left(V_{n}, n \geq 1\right)$ is a sequence of independent zero-mean random vectors in $\mathbb{R}^{m}$. Denote by $r$ the spectral radius of the matrix $A$ and by $p$ the maximal multiplicity of the roots of the minimal polynomial of the matrix $A$ whose the absolute value is $r$. Introduce the following notation:

$$
\begin{gathered}
f_{n}=\sum_{i=1}^{n} \mathbf{E}\left\|V_{i}\right\|^{2} r^{-2 i}\left(1-\frac{i-1}{n}\right)^{2(p-1)}, \quad n \geq 1 ; \\
L_{n}\{f\}=\ln \left(2+\sum_{k=1}^{n-1} \min \left\{M, \ln \left(\frac{f_{k+1}}{f_{k}}\right)\right\}\right), \quad n \geq 2,
\end{gathered}
$$

where $M$ is an arbitrary positive fixed constant and where

$$
\chi_{n}=r^{n} n^{p-1} \sqrt{f_{n} L_{n}\{f\}}, \quad n \geq 2 .
$$

Observe that the sequence $\{f\}=\left(f_{n}, n \geq 1\right)$ is monotone nondecreasing. The bounded law of the iterated logarithm for solutions of equation (13) in the case $\mathbf{E}\left\|V_{n}\right\|^{2} \equiv$ const was studied in [11]. Let us consider a general case.

Theorem 10. Let $\lim _{n \rightarrow \infty} f_{n}=\infty$. Assume that for some $\delta \in(0,1]$ the condition

$$
\sum_{i=1}^{\infty} \sup _{n \geq i}\left[\chi_{n}^{-1}(n+1-i)^{p-1} r^{n-i}\right]^{2+\delta} \mathbf{E}\left\|V_{i}\right\|^{2+\delta}<\infty
$$

holds. Then there exists a nonrandom constant $L \in[0, \infty)$ such that

$$
\limsup _{n \rightarrow \infty} \frac{\left\|Y_{n}\right\|}{\chi_{n}}=L \quad \text { almost surely. }
$$

Proof. Assume that $\operatorname{det} A \neq 0$. Then (13) gives

$$
Y_{n}=A^{n} Y_{0}+A^{n} \sum_{i=1}^{n} A^{-i} V_{i}, \quad n \geq 1
$$

Hence

$$
\chi_{n}^{-1}\left\|Y_{n}\right\| \leq \chi_{n}^{-1}\left\|A_{n}\right\| \cdot\left\|Y_{0}\right\|+\left\|\chi_{n}^{-1} A^{n} \sum_{i=1}^{n} A^{-i} V_{i}\right\|, \quad n \geq 1 .
$$

Observe that

$$
\left\|A^{n}\right\| \leq c_{1}(n+1)^{p-1} r^{n}, \quad n \geq 0,
$$


where $c_{1}$ is a constant independent of $n$, see [3]. By (17) one has

$$
\chi_{n}^{-1}\left\|A^{n}\right\| \cdot\left\|Y_{0}\right\| \underset{n \rightarrow \infty}{\longrightarrow} 0 .
$$

Therefore (15) will be proved if we show that there exists a nonrandom constant $L \in[0, \infty)$ such that

$$
\limsup _{n \rightarrow \infty}\left\|\chi_{n}^{-1} A^{n} \sum_{i=1}^{n} A^{-i} V_{i}\right\|=L \quad \text { almost surely. }
$$

To prove (18), we use Corollary 5 with

$$
A_{n}=\chi_{n}^{-1} A^{n}, n \geq 1 ; \quad X_{i}=A^{-i} V_{i}, i \geq 1 .
$$

Condition (10) follows from (14) by (16). Since (see [5] for more details)

$$
\begin{aligned}
\sum_{i=k+1}^{n}\left\|\chi_{n}^{-1} A^{n} A^{-i} V_{i}\right\|^{2} & \leq c_{1}^{2} \chi_{n}^{-2} \sum_{i=k+1}^{n} r^{2(n-i)}(n+1-i)^{2(p-1)} \mathbf{E}\left\|V_{i}\right\|^{2} \\
& \leq c_{1}^{2}\left(\chi_{n}^{-1} r^{n} n^{p-1} \sqrt{f_{n}}\right)^{2}\left[1-\left(\frac{f_{k}}{f_{n}}\right)^{2}\right]
\end{aligned}
$$

condition (11) holds. Condition (12) also holds for

$$
\varphi_{n}=c_{1}^{2}\left(\chi_{n}^{-1} r^{n} n^{p-1} \sqrt{f_{n}}\right)^{2}, n \geq 1 \text {. }
$$

Since

$$
\chi_{n}^{-1}\left\|A^{n}\right\| \underset{n \rightarrow \infty}{\longrightarrow} 0
$$

Corollary 5 implies that there exists a nonrandom constant $L \in[0, \infty)$ such that

$$
\limsup _{n \rightarrow \infty}\left\|\chi_{n}^{-1} A^{n} \sum_{i=1}^{n} A^{-i} V_{i}\right\|=L \quad \text { almost surely. }
$$

The theorem is proved in the case of $\operatorname{det} A \neq 0$. The proof of the theorem in the case of $\operatorname{det} A=0$ follows similar lines [12].

Corollary 6. Let $r=1$ and $\mathbf{E}\left\|V_{n}\right\|^{2} \equiv$ const. Assume that

$$
\sup _{n \geq 1} \mathbf{E}\left\|V_{n}\right\|^{2+\delta}<\infty
$$

for some $\delta \in(0,1]$. Then there exists a nonrandom constant $L \in[0, \infty)$ such that

$$
\limsup _{n \rightarrow \infty} \frac{\left\|Y_{n}\right\|}{\sqrt{n^{2 p-1} \ln \ln n}}=L \quad \text { almost surely. }
$$

Proof. Since $r=1$ and since $\mathbf{E}\left\|V_{n}\right\|^{2} \equiv$ const, one has

$$
\chi_{n} \sim c_{2} \sqrt{n^{2 p-1} \ln \ln n} \quad(n \rightarrow \infty),
$$

where $c_{2}$ is some positive constant, see [5]. Since for any $i \geq 3$

$$
\sup _{n \geq i}\left[\left(n^{2 p-1} \ln \ln n\right)^{-\frac{1}{2}}(n+1-i)^{p-1}\right]^{2+\delta} \leq \frac{1}{(\ln \ln 3)^{\frac{2+\delta}{2}}} \cdot \frac{1}{i^{1+\frac{\delta}{2}}},
$$


one has

$$
\sum_{i=3}^{\infty} \sup _{n \geq i}\left[\left(n^{2 p-1} \ln \ln n\right)^{-\frac{1}{2}}(n+1-i)^{p-1}\right]^{2+\delta}<\infty .
$$

Thus the condition (14) holds and Corollary 6 is proved.

\section{ACKNOWLEDGMENTS}

The first author is supported by the Ministry of Education and Culture of Spain under grant SAB1998-0169. The authors are grateful to A. Il'enko and V. Zaiats for the help in preparing this article.

\section{REFERENCES}

1. N. N. Vakhania, Probability distributions on linear spaces. Kluwer, Dordrecht, 1981; Russian original: Metsniereba, Tbilisi, 1971.

2. N. N. Vakhania, V. I. Tarieladze, and S. A. Chobanyan, Probability distributions in Banach spaces. Kluwer, Dordrecht, 1987; Russian original: Nauka, Moscow, 1985.

3. V. Buldygin and S. Solntsev, Asymptotic behaviour of linearly transformed sums of random variables. Kluwer, Dordrecht, 1997.

4. V. V. Buldygin and V. O. Koval, The strong law of large numbers for operatornormalized sums of orthogonal random vectors and its application. (Ukrainian) Dopov. Nats. Akad. Nauk Ukr. Mat. Prirodozn. Tekh. Nauki, 1999, No. 10, 11-13.

5. V. O. Koval, Limit theorems for operator-normed sums of random vectors. I, II. Theory Probab. Math. Statist. (to appear).

6. V. V. Buldygin and V. O. Koval, The law of large numbers for vector-valued martingales with matrix normalizations and its applications. (Ukrainian) Dopov. Nats. Akad. Nauk Ukr. Mat. Prirodozn. Tekh. Nauki, 1999, No. 9, 7-11.

7. H. Kaufmann, On the strong law of large numbers for multivariate martingales. Stochastic Process. Appl. 26(1987), No. 1, 73-85.

8. Tze LeUnG LAI, Some almost sure convergence properties of weighted sums of martingale difference sequences. Almost everywhere convergence, II (Evanston, IL, 1989), 179-190, Academic Press, Boston, MA, 1991.

9. A. Bikialis, On estimate of the rest term in the central limit theorem. (Russian) Lith. Math. J. 7(1966), 303-308.

10. V. A. Egonov, On the law of the iterated logarithm. Theory Probab. Appl. 14(1969), 693-699.

11. M. Duflo, Random iterative models. Springer, Berlin, 1997.

12. V. V. Buldygin and V. A. Koval, On the strong law of large numbers for martingales with operator norming. Teor. Sluch. Protses. 4(20)(1998), No. 1-2, 82-88.

(Received 6.08.2000)

Authors' address:

Department of Higher Mathematics No. 1

National Technical university of Ukraine

Kyiv Polytechnic Institute

prosp. Peremohy 37, 02056 Kyiv

Ukraine 